\title{
Poor adherence to parenteral osteoporosis therapies during COVID-19 pandemic
}

\author{
Roland Kocijan $^{1,2,3}$ - Martina Behanova ${ }^{1}$ - Berthold Reichardt ${ }^{4}$. Judith Haschka ${ }^{1,2}$ - Annemarie Kocijan ${ }^{5}$. \\ Jochen Zwerina ${ }^{1,2}$
}

Received: 15 January 2021 / Accepted: 1 February 2021 / Published online: 26 February 2021

(C) International Osteoporosis Foundation and National Osteoporosis Foundation 2021

Keywords COVID-19 · Osteoporosis · Denosumab $\cdot$ Zoledronic acid $\cdot$ Ibandronic acid

\section{Dear Editor,}

We have recently recognized several new cases of multiple vertebral fractures in patients who temporarily discontinued their denosumab (DMAB) therapy. This phenomenon was first described in 2017 [1], but became less apparent in the last years due to increasing awareness as well as education of patients and primary care physicians. The appearance of increasing cases of rebound-associated fractures drew our attention to a possible relationship to the coronavirus disease 2019 (COVID-19) related lockdowns.

The COVID-19 pandemic was first recognized in February 2020 in Austria, followed by a first extensive lockdown in March and April 2020. In that time, there was limited access to outpatient clinics. We present the national data of the DVSV (Dachverband der Sozialversicherungsträger - umbrella association of the health insurance companies including all social security agencies in Austria). Data are based on filled prescriptions for denosumab, zoledronic acid (ZOL), and ibandronic acid (IBA) covering a period from January 2017 until September 2020. The results showed a continuous increase of DMAB prescriptions over the last 2 years with a

Roland Kocijan

roland.kocijan@osteologie.lbg.ac.at

1 Ludwig Boltzmann Institute of Osteology at Hanusch Hospital of OEGK and AUVA Trauma Center Meidling, 1st Medical Department, Hanusch Hospital, Vienna, Austria

2 1st Medical Department, Hanusch Hospital Vienna, Vienna, Austria

3 Medical Faculty of Bone Diseases, Sigmund Freud University, Vienna, Austria

4 Austrian Social Health Insurance Fund, Österreichische Gesundheitskasse, Eisenstadt, Austria

5 Optimal Essen e.U, Vienna, Austria remarkable decrease only during the first COVID-19lockdown in March and April 2020. Thus, a 22 and 23\% reduction as compared with 6 months prior for DMAB prescriptions were observed. Moreover, also the number of prescriptions for (ZOL) was significantly lower in the 2 months of the first lockdown compared with 12 months prior ( -36 and $-49 \%$, respectively). The number of filled prescriptions for intravenous IBA was decreased by 23 and $18 \%$ (when compared with the mean number of filled prescriptions of the prior 12 months) (see Fig. 1).

Bisphosphonates persist in the body for many years [2] and a delay of treatment for 1 or 2 months does not seem to be unfavorable. In contrast, vertebral fractures were reported as early as 8 months since the last DMAB dose [1,3], which can be explained by a rebound increase of bone resorption. A higher number of prescriptions after the first lockdown indicates that numerous patients have received their missed DMAB dose to a later timepoint. However, even a delay of 2 months could increase the risk for rebound-associated vertebral fractures. Therefore, and according to the position statement of the ECTS, patients should continue DMAB treatment or should be switched to an alternative agent [4].

In general, the persistence to DMAB was reported to be consistently high and better compared with other antiosteoporotic agents $[5,6]$. However, physicians are required to continue anti-osteoporotic treatment even in times of COVID-19. Especially patients on Denosumab should receive their treatment on time. That also applies to patients in nursing homes and patients on higher risk for COVID-19 infections. As for rheumatological treatments, patients could be instructed about denosumab self-administration [7]. Telemedicine including telephone and video consultation may increase the adherence to osteoporosis treatments [8]. It is currently unclear if the lower rate of DMAB prescriptions is also associated with a higher rate of rebound-associated 
Fig. 1 Filled drug prescriptions for parenteral osteoporosis therapies during COVID-19 pandemic

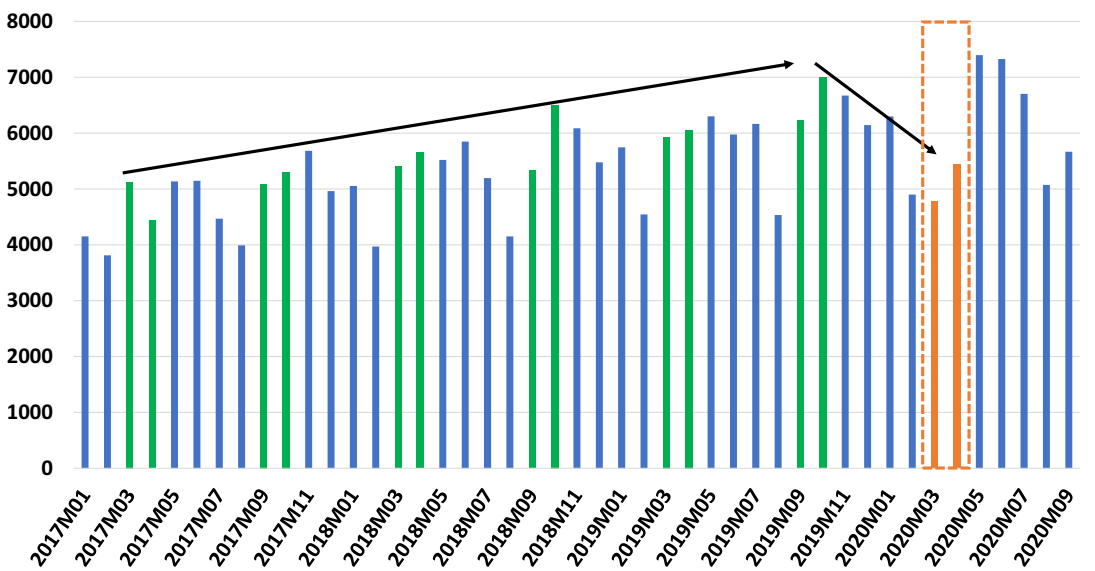

b

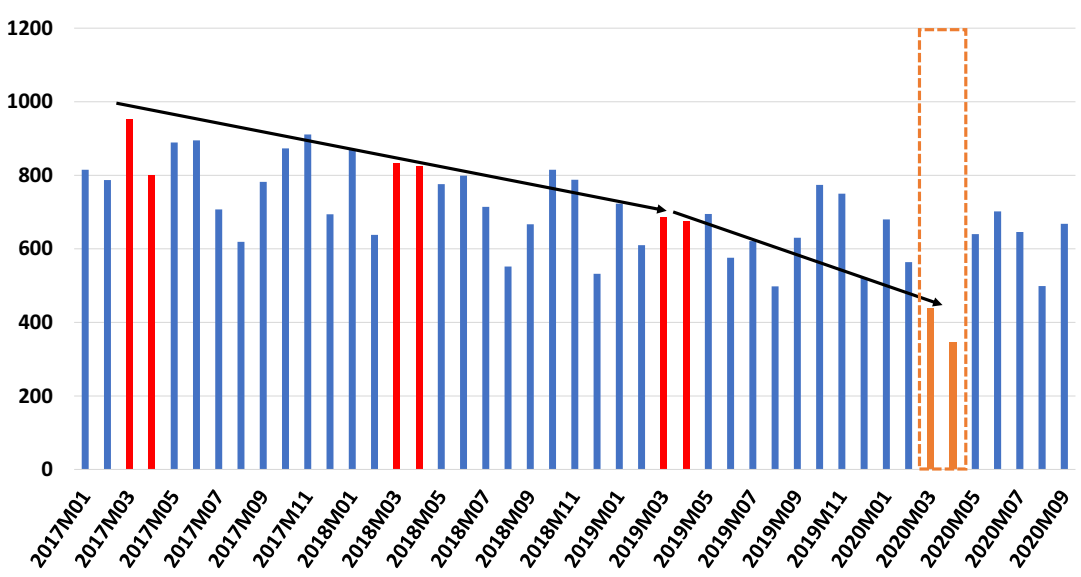

C

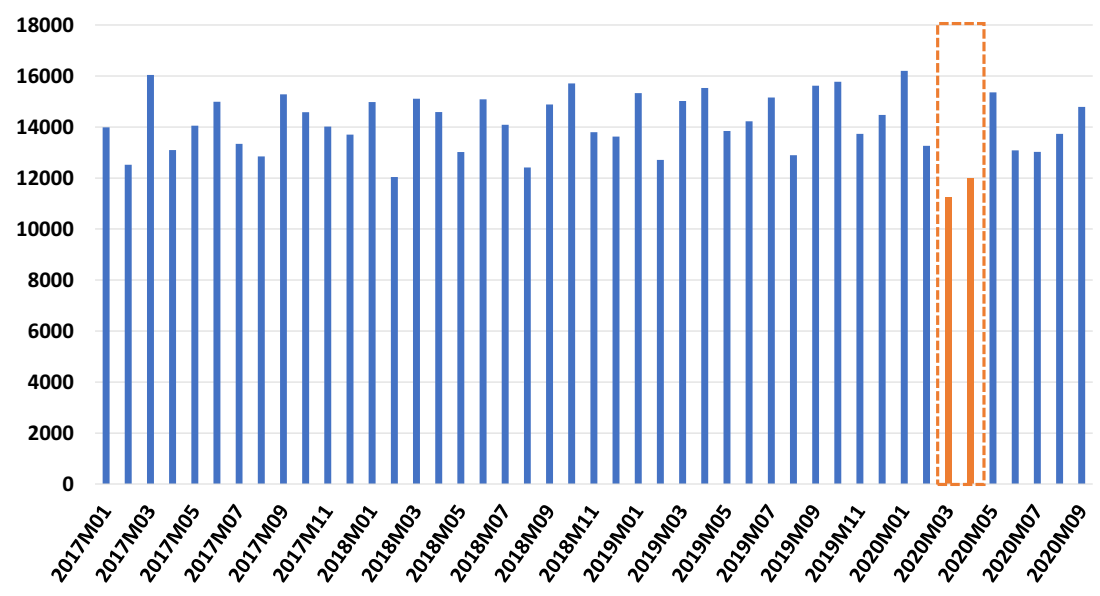

vertebral fractures. Future studies should investigate the impact of repeated lockdowns on DMAB treatment adherence and clinical outcomes.
Orange boxes indicate first COVID-19 lockdown. Green bars indicate the regular interval (6 months) for Denosumab, red bars indicate the regular interval (12 months) for 
zoledronic acid. Total number of filled prescriptions are shown on $\mathrm{Y}$-axis, time period on $\mathrm{X}$-axis.

Funding No funding sources.

Data availability National Data; DVSV, Dachverband der Sozialversicherungsträger

\section{Declarations}

Ethical approval Not applicable

Conflict of interest Roland Kocijan, Martina Behanova, Berthold Reichardt, Judith Haschka, Annemarie Kocijan, and Jochen Zwerina declare that they have no conflict of interest.

\section{References}

1. Anastasilakis AD, Polyzos SA, Makras P, Aubry-Rozier B, Kaouri S, Lamy O (2017) Clinical Features of 24 Patients with reboundassociated vertebral fractures after denosumab discontinuation: systematic review and additional cases. J Bone Miner Res 32(6):12911296

2. Papapoulos SE, Cremers SC (2007) Prolonged bisphosphonate release after treatment in children. N Engl J Med 356(10):1075-1076
3. Anastasilakis AD, Evangelatos G, Makras P, Iliopoulos A (2020) Rebound-associated vertebral fractures may occur in sequential time points following denosumab discontinuation: need for prompt treatment re-initiation. Bone Rep 12:100267

4. Tsourdi E, Langdahl B, Cohen-Solal M, Aubry-Rozier B, Eriksen EF, Guañabens N, Obermayer-Pietsch B, Ralston SH, Eastell R, Zillikens MC (2017) Discontinuation of Denosumab therapy for osteoporosis: A systematic review and position statement by ECTS. Bone 105:11-17

5. Koller G, Goetz V, Vandermeer B, Homik J, McAlister FA, Kendler D, Ye C (2020) Persistence and adherence to parenteral osteoporosis therapies: a systematic review. Osteoporos Int 31(11):2093-2102

6. Fahrleitner-Pammer A, Papaioannou N, Gielen E, Feudjo Tepie M, Toffis C, Frieling I, Geusens P, Makras P, Boschitsch E, Callens J, Anastasilakis AD, Niedhart C, Resch H, Kalouche-Khalil L, Hadji P (2017) Factors associated with high 24-month persistence with denosumab: results of a real-world, non-interventional study of women with postmenopausal osteoporosis in Germany, Austria, Greece, and Belgium. Arch Osteoporos 12(1):58

7. Pal R, Bhadada SK (2020) Managing common endocrine disorders amid COVID-19 pandemic. Diabetes Metab Syndr 14(5):767-771

8. Paskins Z, Crawford-Manning F, Bullock L, Jinks C (2020) Identifying and managing osteoporosis before and after COVID19: rise of the remote consultation? Osteoporos Int 31(9):1629-1632

Publisher's note Springer Nature remains neutral with regard to jurisdictional claims in published maps and institutional affiliations. 